\title{
Progression of peripheral nerve injury in leprosy: Evaluation of the effect of nerve decompression surgery in an endemic region of Brazil
}

\author{
Maria Dias Torres Kenedi ${ }^{a}$, Elifaz de Freitas Cabral ${ }^{b}$, \\ Kazue Narahashi ${ }^{c}$, Silvana Teixeira de Miranda ${ }^{\mathrm{d}}$, \\ Catarina Mabel da Cunha Moreira ${ }^{d}$, Diogo Correia e Silva ${ }^{a}$, \\ Cleumar Silva do Nascimento ${ }^{\mathrm{e}}$, Wanderlei Ruffato ${ }^{\mathrm{f}}$, \\ Antonio José Ledo Alves da Cunha ${ }^{a}$ \& Maria Kátia Gomes ${ }^{a}$ \\ ${ }^{a}$ Rua Professor Rodolpho Paulo Rocco 255, Cidade Universitária, Rio de \\ Janeiro, Federal University of Rio de Janeiro, RJ 21941-913, Brazil \\ ${ }^{\mathrm{b}}$ Surgery, Santa Marcelina Hospital, BR 364, Km 17, Zona Rural, Porto \\ Velho, RO 76801-974, Brazil \\ ' Santa Marcelina Hospital, Dermatology, BR 364, Km 17, Zona Rural, \\ Porto Velho, RO 76801-974, Brazil \\ ${ }^{\mathrm{d}}$ Physiotherapy, Federal University of Rio de Janeiro, Rua Professor \\ Rodolpho Paulo Rocco 255, Cidade Universitária, Rio de Janeiro, RJ \\ 21941-913, Brazil \\ e Santa Marcelina Hospital, BR 364, Km 17, Zona Rural, Porto Velho, RO \\ 76801-974, Brazil \\ ${ }^{\mathrm{f}}$ Oswaldo Cruz Polyclinic, Av. Gov. Jorge Teixeira 3862, Industrial, \\ Porto Velho, RO 76821-096, Brazil
}

Submitted 19 January 2021; Accepted 22 April 2021

\begin{abstract}
Summary
Aim: To evaluate the progression of nerve injury in leprosy patients undergoing peripheral nerve decompression surgery.

Methodology: Observational study of retrospective, descriptive-analytical cohort. In the convenience sample, individuals with leprosy who underwent peripheral nerve decompression surgery at the Casa de Saúde Santa Marcelina, Porto Velho/RO, between 2000 and 2019 were evaluated nerve by nerve, before and after surgery.

Results: Note that 574 individuals underwent 2549 surgeries of the ulnar, median, tibial and/or fibular nerves. Most individuals were male (51.6\%), of brown race (80.3\%), multibacillary $(90.9 \%)$, aged between 31 and 60 years $(73.2 \%)$ and originating from other municipalities in the state of Rondônia, Northern Region of Brazil (67.4\%). Most individuals maintained or improved the GD (WHO Grade of Disability), the sensory or the motor nerve function in the limbs operated.
\end{abstract}

Correspondence to: Maria Dias Torres Kenedi (e-mail: mdtkenedi@yahoo.com.br) 
Conclusion: The improvement or maintenance of the DG was observed in more than $80 \%$ of the operated individuals. In the nerve-to-nerve analysis, considering the sensory and motor functions for all operated nerve trunks, there was an improvement or maintenance of the values presented in the preoperative period for most individuals. According to the results obtained in this study, we concluded that peripheral nerve decompression surgery was able to interrupt the progression of nerve injury in more than $80 \%$ of the operated individuals. Considering the potential of the surgery as a preventive procedure, we recommend its indication even for individuals with grade 0 of disability but that present persistent pain or neural thickening after four weeks of clinical treatment without other symptoms.

Keywords: Leprosy, neurolysis, neuropathy

\section{Introduction}

Despite all the efforts to detect and control leprosy, Brazil continues to occupy the second position in number of cases of the disease in the world, thus being responsible for more than 93\% of all cases diagnosed in the Americas in the last year. ${ }^{1,2}$ In 2019, 27,864 new cases of leprosy were registered in Brazil, thus resulting in a new case detection rate of 1.50/10,000 inhabitants. ${ }^{1,2}$

In the same year, the state of Rondônia presented 465 new cases of the disease, corresponding to a new case detection rate of 3.83/10,000 inhabitants, the sixth highest in the country, i.e., well above the national rate for the same year and the goal recommended by the World Health Organization (WHO), which is $1 / 10,000$ inhabitants. $^{3}$

The burden of leprosy is related to the progression of nerve injury, which can occur even after treatment with the specific multidrug therapy (MDT), thus causing physical disability with consequences at work and in the social life. Peripheral nerve decompression surgery is a complementary therapy to conservative clinical treatment with systemic corticosteroids recommended by the World Health Organization (WHO). The prolonged use of corticosteroid therapy does not seem to interrupt the progressive course of nerve injury and can lead to serious adverse effects such as glaucoma, diabetes and systemic arterial hypertension. Moreover, corticosteroids are often misindicated in the management of chronic pain. Peripheral nerve decompression surgery is considered a preventive surgery for nerve injury in leprosy and when properly indicated; it aims to prevent the progression of nerve injury, avoiding the emergence of new physical disabilities.

The involvement of peripheral nerve trunks in leprosy leads to an increase in their volume, because of an inflammatory reaction either by direct aggression of the bacillus or indirectly by the individual's own immune system. This causes its compression by the osseofibrous tunnels that surround them. Neurolysis reduces this extrinsic compression, thus allowing the improvement of neural circulation, which favors the local effect of the corticoid and limits the progression of nerve injury. This surgery can reduce the time of corticoid use and its adverse effects; however, we believe that its primary worth is to prevent the progression of nerve injury in leprosy.

For the National Leprosy Control Program, innovative strategies are essential in addressing acute and chronic neuritis, which aim to reduce the time of corticosteroids use, thus interrupting the progression of nerve injury and maintaining work function and the social participation of the patient. Therefore, it is essential that endemic countries such as Brazil discuss the role 
of peripheral nerve decompression surgery as one of the routine procedures of leprosy control programs, primarily because of its potential to prevent those individuals diagnosed already with nerve injury from presenting progression of physical disabilities, which impair their social insertion and their functional and work capacity.

This study aims to investigate the results of a cohort of patients who underwent surgical decompression of peripheral nerves to support the operationalization of this procedure in the different states of Brazil. For this purpose, the effect of peripheral neural decompression surgery on individuals with leprosy with regard to the progression of neural damage was studied. Other aims of this study were as follows: to evaluate the WHO grade of disability of individuals undergoing peripheral nerve decompression surgery before and after the surgical procedure, thus considering limb by limb; to analyze nerve by nerve the evolution of nerve injury in individuals with leprosy undergoing peripheral nerve decompression surgery; to compare the impairment of each nerve operated before and after the surgical procedure; to evaluate whether nerve decompression surgery interrupts the progression of nerve injury in individuals with leprosy; to identify the influence of sociodemographic and clinical factors on the progression of nerve injury in leprosy and to analyze possible associations between the categorical and continuous variables of interest with the data of physical and functional evaluation.

\section{Methods}

Observational study of retrospective, descriptive-analytical cohort to evaluate the effect of peripheral nerve decompression surgery on the evolution of nerve injury in leprosy.

The surgeries were performed using the techniques recommended by the Brazilian Ministry of Health and the Simplified Neurological Assessment examination was performed as part of the pre- and postoperative procedures. All assessments were carried out by the same examiner, an expert in the performance of the evaluation with the Simplified Neurological Assessment examination form and regularly trained by the Ministry of Health of Brazil. Data was later collected directly from the patients' medical records.

The study site was the Casa de Saúde Santa Marcelina (CSSM), in Porto Velho, Rondônia, a referral hospital for preventive and reparative surgeries and rehabilitation of individuals with leprosy for the entire Northern Region of Brazil.

Individuals who underwent peripheral nerve decompression in one or more of the following nerves: ulnar and median in the upper limbs as well as tibial and fibular in the lower limbs between 2000 and 2019 were included. Each individual may have had one to eight nerves operated. The eligibility criteria for surgery were as follows: patients diagnosed with leprosy paucibacillary $(\mathrm{PB})$ or multibacillary $(\mathrm{MB})$ who have: recurrent neuropathy despite the use of corticosteroids; nerve abscess; neuropathy that does not respond to systemic corticosteroid therapy for a period of four weeks; tibial nerve neuropathy; patients with contraindications to the use of corticosteroids; chronic neuropathy with late deficit and pain; progressive worsening of nerve injury, verified through tests of nerve function by the Simplified Neurological Assessment examination. ${ }^{4}$ Note that the surgical technique used in all patients did not vary over the study period. The surgery consisted of the external decompression and release of the nerves in situ. For ulnar nerve surgery, its anterior transposition and subfascial protection were performed for all individuals operated. Due to the extensive fibrosis involved on leprosy neuropathy which impair neural mobility our experience reveals that the anterior transposition of the ulnar nerve increases its mobility and helps to avoid pain after surgery. In our practice, the presence of nerve enlargement does not mean that the epineurium has to be opened. It is 
realized only in cases of neural abscess, which did not happen on the studied subjects. Our experience shows that there was no case of ulnar luxation after surgery in the individuals operated.

The following were excluded from this study: medical records without pre or postoperative evaluations; surgeries with postoperative evaluation less than a month after the procedure; individuals whose single evaluation was performed on the day of surgery and surgeries performed on the date of the last evaluation (other previous surgeries on the same individual were considered). The Simplified Neurological Assessment data regarding the eyes and the radial nerve in the upper limbs were not included in the analysis because they are outside the scope of this study.

For analysis, we considered the dates prior to surgery closest to the day of the procedure and the last post-surgical evaluation; however, at least one month after the operation. This period was considered sufficient to observe symptom improvement, based on the results of other authors. 5

The physical examination reported in the medical records comprised the patient's sensorymotor assessment through the Simplified Neurological Assessment examination. Data regarding the patient's sensory function were obtained through the evaluation with the SemmesWeinstein monofilaments. The muscle strength was tested using the Kendall's manual resistance test. ${ }^{6}$ For examining muscular strength, the following movements were evaluated for each nerve: ulnar-abduction of the 5th finger; median-abduction of the thumb; fibulardorsal flexion of the ankle and extension of the hallux. Intrinsic muscles for hands and feet were tested as a whole by the test of the intrinsic position of the hands and the evaluation of the presence or not of claws in hands and feet.

Based on the result of these tests, the grade of physical disability (DG) was obtained: individuals without sequelae have DG 0 , individuals with impairment in sensitivity or strength in the feet and/or in the hands have DG 1 and those with visible changes resulting from the disease present DG 2.4,7,8 Following neural evaluation, those who presented the same value for strength and sensitivity before and after surgery were considered as stationary/not worsening (not progressed of neural impairment); those with a biggest value were considered as improvement and those with smaller values for those tests were considered as worsening. For the disability grade, those who kept the same grade after surgical procedure were considered stationary/not worsening (not progressed of neural impairment), those who reduced the grade were considered as improvement and those who increased the value of the DG were considered as worsening. Note that we considered improvement and stationary cases (not worsening) as not progression of peripheral nerve injury.

The DG can be analyzed as an absolute value for the patient or by the sum of the degree attributed to each evaluated segment: eyes, right and left, hands and feet. This is the EHF (EyeHand-Foot) score and is considered more sensitive to assess the disability of leprosy patients than the DG because it allows to identify the segment(s) affected and can vary between 0 and 12. In this study, the maximum value considered was 8 because the eyes were not analyzed.

The sociodemographic, clinical, surgical and neurological assessment data were stored in a Microsoft Excel for Mac 2011 spreadsheet and later transferred for analysis with the IBM SPSS statistics software, version 26.

A descriptive analysis of the clinical and sociodemographic data was performed. The categorical variables were analyzed using frequency distribution and the numerical variables with the main central tendency and dispersion statistics: mean and standard deviation. Moreover, the $95 \%$ confidence interval for the main parameters was calculated. The statistical significance for 
analyzing DG, strength and sensitivity before and after surgery was obtained using Wilcoxon's test. To compare the DG of each limb before and after surgery, the Test $\chi^{2}$ by McNemarBowker was used. For analyzing the association between DG improvement and worsening with the variables sex, race, age range, operational classification and clinical form, the test $\chi^{2}$ was used. The Spearman's correlation coefficient was used to analyze the correlation between the MDT time and the surgery. To study the factors associated with the worsening or not worsening of the DG, the multiple logistic regression was performed incorporating the following variables: operational classification, sex, clinical form, MDT time and neurolysis time. For all the analyses, a 95\% confidence interval and a level of statistical significance of $p$ $<0.05$ were adopted.

\section{Results}

Note that 623 individuals registered as leprosy patients who underwent peripheral nerve decompression surgery were selected. Of these, 47 individuals were excluded because the date of the single surgery performed coincided with the date of the last evaluation and two patients were excluded because of the lack of evaluation in the medical record. The final sample included 574 operated individuals. Among them, 60 surgeries were excluded because they coincided with the last evaluation; however, other surgeries performed on the same individuals were considered. The final sample had a total of $1305 \mathrm{limbs}$ and 2549 surgeries performed. The distribution of the sample by gender was quite balanced, comprising 296 individuals $(51.6 \%)$ male and $278(48.4 \%$ ) female, being $79.8 \%$ of them of brown race. Only $31.7 \%$ of individuals evaluated resided in Porto Velho (182 individuals), most were from other municipalities in the state of Rondônia (67.4\%). The average age at the date of the first surgery of the individuals evaluated was 42.87 years and $73.2 \%$ of them were in the age range from 31 to 60 at the date of the first surgery (420 individuals). Regarding occupation, $28 \%$ of individuals had rural activities and $22.6 \%$ were housewives (Table 1).

Note that more than $90 \%$ of the individuals studied were multibacillary (MB), being the vast majority composed of the dimorphic clinical form (84.7\%). Of the 574 operated individuals, $122(21.3 \%)$ had the four limbs operated and $178(31.0 \%)$ had only one limb operated. To summarize, 2549 surgeries were performed, distributed in 1305 limbs (Table 2).

The time elapsed between the MDT and the surgery was similar between all limbs and did not showed any correlation with the DG in the last evaluation according to the Spearman's correlation coefficient (Figure 1).

Among the 574 individuals evaluated, 247 were reclassified from DG 0 to DG 1 on one or more evaluation maps because of the change in classification that occurred in 2016, which started to consider as DG 1 the individuals with strength less than 5. The degree of physical disability (DG) was analyzed limb by limb, thus comparing the value of each limb before surgery and in the last evaluation performed. The same was performed with respect to the sensitivity and the strength of each operated limb (Tables 3-5).

The improvement or worsening in the DG was not associated with the clinical and sociodemographic variables: sex, race, age range, operational classification and clinical form for the four limbs (Table 6).

The analysis by logistic regression demonstrated that only the time elapsed between surgery and the last evaluation showed a statistically significant correlation with the worsening or not worsening of the DG for the RSL ( $p=0.10$ ) and for the LSL (0.009). The analysis of the EHF (eye-hand-foot) score demonstrated that $363(63.24 \%)$ of the total of 574 operated individuals maintained the EHF score better or equal to that presented before surgery. When observing 
Table 1. Sociodemographic data of the 574 individuals evaluated

\begin{tabular}{lc}
\hline Characteristics & $n(\%)$ \\
& $n=574$ \\
\hline Gender & \\
Female & $278(48.4)$ \\
Male & $296(51.6)$ \\
Age range & \\
Up to 30 years & $115(20.0)$ \\
From 31 to 60 years & $420(73.2)$ \\
Above 61 years & $39(6.8)$ \\
Race & \\
Black & $32(5.6)$ \\
Brown & $461(80.3)$ \\
White & $81(14.1)$ \\
Origin & \\
Porto Velho & $182(31.7)$ \\
Other municipalities-RO & $387(67.4)$ \\
Other states & $4(0.7)$ \\
Uninformed & $1(0.2)$ \\
Occupation & \\
Farmer (rural activities) & $161(28)$ \\
Housewife & $130(22.6)$ \\
General services & $29(5.1)$ \\
Student & $12(2.1)$ \\
Bricklayer & $20(3.5)$ \\
Retired & $23(4.0)$ \\
Others & $188(32.8)$ \\
Uninformed & $11(1.9)$ \\
\hline
\end{tabular}

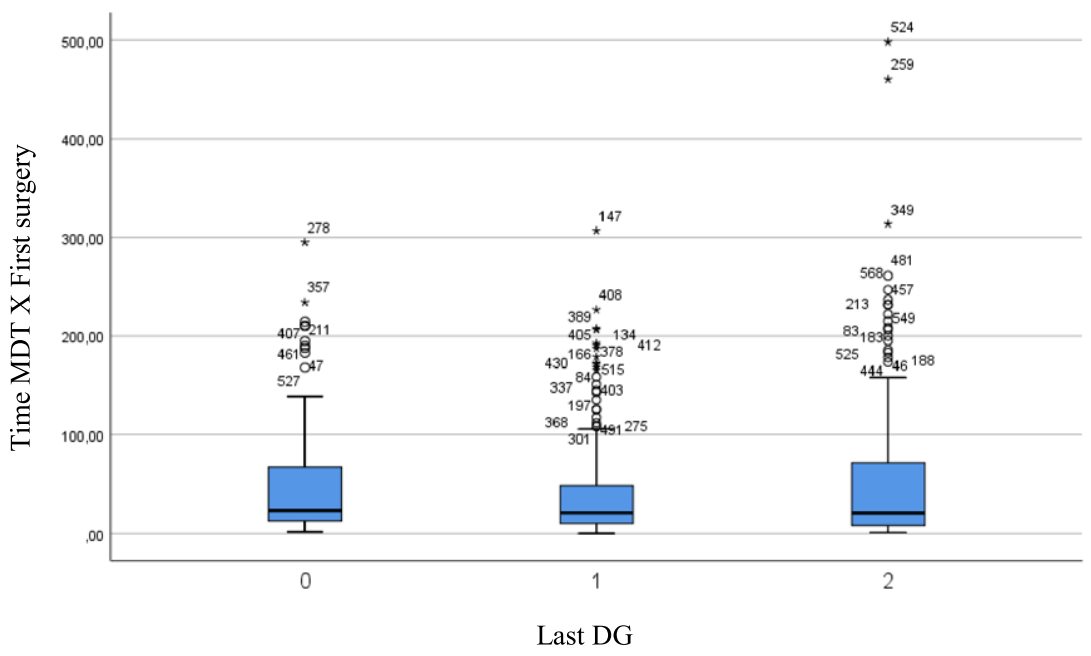

Figure 1. Relationship between the time elapsed between MDT and the first surgery and the total DG of the operated individuals.

the EHF score of the 122 individuals who underwent surgery of the four limbs, it is clear that $59.01 \%$ (72 individuals) maintained or improved the DG after surgery. 
Table 2. Clinical and surgical data of the 578 individuals evaluated

\begin{tabular}{|c|c|}
\hline Characteristics & $\begin{array}{c}n(\%) \\
(n=574)\end{array}$ \\
\hline \multicolumn{2}{|l|}{ Operational rating } \\
\hline MB (multibacillary) & $522(90.9)$ \\
\hline PB (paucibacillary) & $52(9.1)$ \\
\hline \multicolumn{2}{|l|}{ Clinical form } \\
\hline TL (tuberculoid leprosy) & $34(5.9)$ \\
\hline DL (dimorphic leprosy) & $486(84.7)$ \\
\hline VL (virchowian leprosy) & $54(9.4)$ \\
\hline \multicolumn{2}{|l|}{ Number of operated limbs/patient } \\
\hline 1 & $178(31.0)$ \\
\hline 2 & $167(29.1)$ \\
\hline 3 & $107(18.6)$ \\
\hline 4 & $122(21.3)$ \\
\hline No of operated nerves & Number of surgeries performed \\
\hline RSL (294) Ulnar & 289 \\
\hline Median & 292 \\
\hline LSL (285) Ulnar & 283 \\
\hline Median & 282 \\
\hline RIL (363) Tibial & 363 \\
\hline Fibular & 347 \\
\hline LIL (363) Tibial & 361 \\
\hline Fibular & 332 \\
\hline Total $(1305)$ & 2549 \\
\hline Average time between surgery and last assessment & (In months) \\
\hline RSL & 57,2 \\
\hline LSL & 62,9 \\
\hline RIL & 65,2 \\
\hline LIL & 66,0 \\
\hline
\end{tabular}

Abbreviations: RSL—right superior limb; LSL_left superior limb; RIL—right inferior limb; LIL—left inferior limb.

Table 3. Disability Grade: improvement $\times$ worsening

\begin{tabular}{cccc}
\hline Nerve & Improvement $n(\%)$ & Without change $n(\%)$ & Worsening $n(\%)$ \\
\hline RSL $(n=294)$ & $\mathbf{4 7}(\mathbf{1 6 . 0})$ & $\mathbf{2 1 4}(\mathbf{7 2 . 8})$ & $33(11.2)$ \\
LSL $(n=285)$ & $\mathbf{5 1}(\mathbf{1 7 . 9})$ & $\mathbf{1 8 6}(\mathbf{6 5 . 3})$ & $48(16.8)$ \\
RIL $(n=363)$ & $\mathbf{6 5}(\mathbf{1 7 . 9})$ & $\mathbf{2 3 7}(\mathbf{6 5 . 3})$ & $61(16.8)$ \\
LIL $(n=364)$ & $\mathbf{6 2}(\mathbf{1 7 . 0})$ & $\mathbf{2 4 5}(\mathbf{6 7 . 3})$ & $57(15.7)$ \\
\hline
\end{tabular}

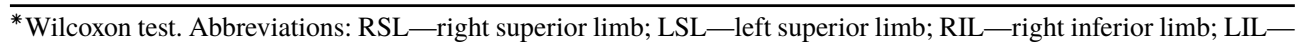
left inferior limb.

\section{Discussion}

Our results indicate that more than $80 \%$ of individuals operated at the Casa de Saúde Santa Marcelina (88.8\% of the RSL, $83.2 \%$ of the LSL, $83.2 \%$ of the RIL and $84.3 \%$ of the LIL) had maintenance or improvement of DG after surgery, regardless of the time between diagnosis and surgical treatment.

The Casa de Saúde Santa Marcelina, in Porto Velho, Rondônia, is the primary reference center in the Northern Region of Brazil for corrective and reparative surgeries in leprosy and serves individuals from several municipalities in the state, as well as other states in the Northern Region. 
Table 4. Improvement $\times$ worsening of sensitivity

\begin{tabular}{lccrc}
\hline Nerve & Improvement $n(\%)$ & Without change $n(\%)$ & Worsening $n(\%)$ & $p$-value \\
\hline R Median $(n=294)$ & $\mathbf{6 1}(\mathbf{2 0 . 7})$ & $\mathbf{1 5 0}(\mathbf{5 1 . 1})$ & $83(28.2)$ & 0.025 \\
R Ulnar $(n=294)$ & $\mathbf{8 4}(\mathbf{2 8 . 6})$ & $\mathbf{1 1 6}(\mathbf{3 9 . 5})$ & $94(32.0)$ & 0.527 \\
R Fibular $(n=363)$ & $\mathbf{1 0 1}(\mathbf{2 7 . 8})$ & $\mathbf{1 4 9}(\mathbf{4 1 . 0})$ & $113(31.1)$ & 0.398 \\
R Tibial $(n=363)$ & $\mathbf{1 5 0}(\mathbf{4 1 . 3})$ & $\mathbf{4 4}(\mathbf{1 2 . 1})$ & $169(46.6)$ & 0.041 \\
L Median $(n=285)$ & $\mathbf{5 6}(\mathbf{1 9 . 6})$ & $\mathbf{1 4 1}(\mathbf{4 9 . 5 )}$ & $88(30.9)$ & 0.019 \\
L Ulnar $(n=285)$ & $\mathbf{8 9}(\mathbf{3 1 . 3})$ & $\mathbf{1 1 1}(\mathbf{3 8 . 9})$ & $85(29.8)$ & 0.094 \\
L Fibular $(n=364)$ & $\mathbf{7 8}(\mathbf{2 1 . 4})$ & $\mathbf{1 7 0}(\mathbf{4 6 . 7})$ & $116(31.9)$ & $\mathbf{0 . 0 0 6}$ \\
L Tibial $(n=364)$ & $\mathbf{1 4 2}(\mathbf{3 9 . 0})$ & $\mathbf{5 5}(\mathbf{1 5 . 1})$ & $167(45.9)$ & 0.055 \\
\hline
\end{tabular}

${ }^{*}$ Wilcoxon test.

Table 5. Improvement $\times$ worsening of muscle strength

\begin{tabular}{lcccc}
\hline Nerve & Improvement $n(\%)$ & Without change $n(\%)$ & Worsening $n(\%)$ & $p$-value* \\
\hline R Median $(n=294)$ & $\mathbf{3 3}(\mathbf{1 1 . 2})$ & $\mathbf{2 0 3}(\mathbf{6 9 . 0})$ & $58(19.7)$ & 0.013 \\
R Ulnar $(n=294)$ & $\mathbf{6 8}(\mathbf{2 3 . 1})$ & $\mathbf{1 4 5}(\mathbf{4 9 . 3})$ & $81(27.6)$ & 0.243 \\
R Fibular $(n=363)$ & $(1) \mathbf{5 4}(\mathbf{1 4 . 9})$ & $\mathbf{2 3 7}(\mathbf{6 5 . 3})$ & $72(19.8)$ & 0.072 \\
& $(2) \mathbf{4 2}(\mathbf{1 1 . 6})$ & $\mathbf{2 6 8}(\mathbf{7 3 . 8})$ & $53(14.6)$ & 0.383 \\
L Median $(n=285)$ & $\mathbf{3 8}(\mathbf{1 3 . 3})$ & $\mathbf{1 8 9}(\mathbf{6 6 . 3})$ & $58(20.4)$ & 0.057 \\
L Ulnar $(n=285)$ & $\mathbf{6 8}(\mathbf{2 3 . 9})$ & $\mathbf{1 3 9}(\mathbf{4 8 . 8})$ & $78(27.4)$ & 0.202 \\
L Fibular $(n=364)$ & $(1) \mathbf{5 0}(\mathbf{1 3 . 7})$ & $\mathbf{2 2 9}(\mathbf{6 2 . 9})$ & $85(23.4)$ & $<0.001$ \\
& $(2) \mathbf{3 8}(\mathbf{1 0 . 4})$ & $\mathbf{2 6 1}(\mathbf{7 1 . 7})$ & $65(17.9)$ & 0.038 \\
\hline
\end{tabular}

(1) Hallux extension; (2) plantar flexion.

Table 6. Statistical significance of sociodemographic and clinical variables

\begin{tabular}{lcccc}
\hline Variable & \multicolumn{3}{c}{$p$-value } & LIL \\
\cline { 2 - 5 } & RSL & LSL & 0.410 & 0.910 \\
Gender & 0.273 & 0.431 & 0.703 & 0.415 \\
Race & 0.458 & 0.565 & 0.861 & 0.282 \\
Age range & 0.793 & 0.287 & 0.834 & 0.578 \\
Operational & 0.443 & 0.032 & 0.217 & 0.616 \\
classification & & & & 0.090 \\
Clinical form & 0.621 & 0.090 & \\
\hline
\end{tabular}

${ }^{*}$ Test $\chi^{2}$. Abbreviations: RSL—right superior limb; LSL—left superior limb; RIL—right inferior limb; LIL—left inferior limb.

The recommendation of Brazilian Ministry of Health is that patients should be referred to surgery in case of lack of improvement of the symptoms after four weeks of corticoid therapy at the first episode of neuritis. ${ }^{9}$ However, in our sample, patients were referred to surgery after two or three episodes of neuritis without improvement with corticotheraphy. One reason for that delay may be the distance between these municipalities and the hospital. Patients are often referred for surgery when all other resources have failed to control the progression of neuropathy, and therefore already have some degree of established nerve injury. Our recommendation, according with other authors, is that individuals should be operated on as soon as possible to avoid the progression of neural damage. ${ }^{10}$ We observed, however, that even in cases where the surgical procedure was performed late, it was effective in containing the progression of the nerve injury, acting as a preventive for installing more serious and disabling sequelae, even when there is already certain neural involvement. 
Malaviya, in his classic 2004 article, "Shall we continue with nerve trunk decompression in leprosy?" discussed peripheral neuropathies in leprosy. He reported a tendency to compare the results of drug treatment (corticotherapy) and surgical treatment (decompression of nerve trunks) as if these methods competed with each other when, in fact, they should be considered as complementary techniques. ${ }^{11}$

According to Sette et al., the early neurolysis is an alternative therapy that should not be neglected and can be concurrently performed with the administration of corticosteroids, particularly in young patients at risk for functional sequelae or significant nerve involvement. ${ }^{12}$

According to Malaviya, the time between the diagnosis of neuropathy and the surgery is essential for the good result of the procedure. ${ }^{11}$ In our study, as most of our sample comes from other municipalities or states $(68.1 \%)$, we do not know the date of the onset of neuropathy; however, we can say that the time between the start of treatment with MDT and the performing of the surgeries had no significant impact on the results of the procedure.

In the sample studied in this research, 486 individuals $(84.7 \%$ ) were DL (dimorphic leprosy). Because of this immunological instability and the increased risk of developing neuropathies, many of these individuals have an indication for preventive surgery in all nerve trunks, even when they still have grade 0 of physical disability. These grade 0 individuals may have neuropathic pain and neural thickening, even in the absence of sensory or motor symptoms. These factors are suggestive of neural involvement and corroborate the indication for surgery for this group. In the sample studied, 109 individuals had DG 0 in all limbs before the first surgery $(\mathrm{EHF}=0)$ and 118 remained without disabilities after the last evaluation.

When the nerve is enlarged, the effect of the corticotherapy is impaired by the significative mechanical factor of the external compression. In these cases, we believe that early surgery will be more effective in preventing disability than the clinical treatment only, even when symptoms are not present yet (GD0). This reinforces the importance of the regular examination of those patients with increased risk of neuropathy through the neural evaluation ever three months during MDT or at any time when the individual refers worsening of the symptoms, as recommended by Brazilian Ministry of Health.

Although leprosy is a slowly progressing disease, the leprosy reactions are common and can result in rapid progression of neurological deficits and permanent disability. ${ }^{13}$ Note that about $30 \%$ of the individuals after discharge from MDT are subject to immunological reactions that can determine additional damage to the peripheral nerve. ${ }^{14}$ Among these individuals, those of the dimorphic clinical form (DL) stand out who, because of their immunological instability, are more predisposed to reaction states and the consequent prolonged use of corticosteroid therapy and its adverse effects.

Of the total number of individuals studied, 522 individuals $(90.9 \%)$ were MB, most of them dimorphic. This represents an initial risk of developing nerve injury of at least $16 \%$ during or after MDT, according to the predictive factors of Croft et al. (2000), regardless of other factors. ${ }^{15}$ The current recommendations for follow-up and monitoring of individuals diagnosed only during MDT can leave a large proportion of individuals who may develop neuropathies and consequent disabilities after discharge without necessary interventions. Rodrigues et al. observed a high percentage of MB individuals (86\%), and $92.1 \%$ of the their total sample had some degree of disability at the time of the diagnosis. ${ }^{16}$

Reis (2012) cites as risk factors for nerve injury the most advanced age, the male gender, the multibacillary form, several nerve trunks thickened at the time of the diagnosis, occurrence of reverse reactions, degree of disability and the high bacterial burden, as demonstrated in the 
literature. ${ }^{17-19}$ In our sample, 51.6\% (296 individuals) were male and 90.9\% (522 patients) were multibacillary, which includes them in the high-risk group for developing neuropathies.

It is worth mentioning that this is the study performed with the highest number of surgeries performed (2549), among all studies consulted. According to Mendes, the interpretation of the surgical results must be careful considering that the functional recovery of the nerve depends on the clinical form of the disease, the age of the patient, the duration time of the neuritis, the extent of the extrinsic compression, immunological status of the patient and the effectiveness of drug treatment. Patients who have a shorter duration of symptoms and fewer painful crises show better recovery in the postoperative period. ${ }^{10}$

The findings of improvement or stabilization of sensory or motor impairment in the operated nerves have been reported in other studies in a similar proportion to those of our research. Husain et al. (2003) analyzed 59 ulnar and 22 median nerves in PB individuals with complaints of impairment of these nerves, symptomatic or not, and reported that, of the symptomatic individuals who were operated, one third improved, another third maintained the condition after surgery and another third showed worsening of neural function, despite the association of the surgical procedure with corticotherapy, i.e., in two thirds of the sample they studied, there was no progression of nerve injury.

In our study, more than $80 \%$ of the operated individuals showed improvement or stabilization of the nerve injury. Some of the asymptomatic and nonoperated patients in the study by Husain et al. showed worsening during MDT, even when they had normal clinical and electromyographic exams, which corroborates the importance of knowing the individual's immune profile and shows how complex it is to determine more sensitive predictive factors for the early indication of medical or surgical treatment. Moreover, PB individuals such as those in the study tend to have an abrupt worsening of the symptoms, which makes the preventive nature of the surgery an important fact to be considered when there is any indication of nerve involvement. Note that all individuals who were operated in the study by Husain et al. showed improvement of pain, even those who showed no improvement or worsening of the sensory and motor conditions. ${ }^{20}$

Tests such as electroneuromyography (ENMG), as well as specific genetic or immunological tests are not yet available in most services that accompany these patients; hence, the importance of Simplified Neurological Assessment in the routine of the services in a systematic manner.

In another study, Husain et al. followed 193 individuals who had ulnar nerve operated between 1982 and 1992 and observed sensory improvement in 48.7\% of the cases. Motor improvement occurred slowly for up to two years. Of the patients followed for a period of 3-10 years, $45.1 \%$ showed remarkable motor improvement and 45\% maintained acceptable muscle function for performing activities of daily living (ADL). ${ }^{21}$ According to Husain (2013), the musculature innervated by the ulnar maintains its potential to prevent deformities when it presents a graduation equal to or $>3$. In this study, Husain (2013) reports $100 \%$ improvement of the pain and of the strength in $50 \%$ of operated ulnar nerves. The median and tibial nerves also had promising results and $100 \%$ of the plantar ulcers healed after decompression of the tibial (ulnar and median sensitivity improved in $48 \%$ of the cases and $50 \%$ of the individuals had grade 5 of strength after surgery). ${ }^{5}$

In our sample, $74.3 \%$ of the individuals who operated the RSL and $55.5 \%$ of those who operated the LSL had grade 3 of strength or more for the ulnar musculature after the surgery, and therefore preserved the ability of this musculature to maintain the balance of the hand structure, thus avoiding the formation of claws. Although dominance was not controlled in this study, we believe that it may be related to the best result of RSL. 
The decompression of the tibial nerves helps to release the pressure in the neurovascular complex, which overcomes the problem of vascular ischemia and improves the vascular supply of the foot sole. This decompression helps to cure plantar ulcer and prevents its recurrence. ${ }^{5}$ In our sample, only $4.1 \%$ of the RIL and $5.2 \%$ of the LIL had plantar ulcer in the postoperative period. Of individuals who had ulcer before RIL surgery, $75 \%$ had wound healing in the last evaluation. In the LIL, $57.7 \%$ of the operated individuals had their ulcers healed. The same research group is performing a study about the follow-up of these patients after surgery and the healing of the ulcers. These results will be available soon.

It can be inferred that the progression of nontrunk pathology, such as arthropathy, joint and muscle degeneration, occurs over time because of injury that has already occurred and not because of the progressive worsening of the peripheral nerve. This may justify the progression of the degree of disability in certain individuals undergoing surgery.

As a limitation of this study, we can mention the lack of clinical data the time of the diagnosis of most of the sample, which made it impossible to assess the evolution of the individuals between the diagnosis and the first surgery.

Another study is being conducted by our research team to compare our results with the experience of other centers where the surgery is not performed for operational reasons.

\section{Conclusions}

An improvement or maintenance of the DG was observed in more than $80 \%$ of the operated individuals. In the nerve-to-nerve analysis, considering the sensory and motor functions for all operated nerve trunks, there was an improvement or maintenance of the values presented in the preoperative period for most individuals. According to the results obtained in this study, we concluded that peripheral nerve decompression surgery was able to interrupt the progression of nerve injury in more than $80 \%$ of the operated individuals. Considering the potential of the surgery as a preventive procedure, we recommend its indication even for individuals with grade 0 of disability but that present persistent pain or neural thickening after four weeks of clinical treatment without other symptoms.

\section{Acknowledgements}

We are grateful to Professor Ronir Raggio, PHD for the assistance in the statistical analysis and to the librarian Vanessa Mendonça for the support with the search for articles.

We also thank the Casa de Saúde Santa Marcelina team for the support to the realization of this research.

\section{Ethics approval}

This research was approved by the Research Ethics Committee of HUCFF-UFRJ under CAAE 38240220.8.0000.5257. Data collection only started after its approval by this committee.

\section{Competing interests}

All authors declare that the answer to the question on competing interest forms are 'No', and therefore have nothing to declare.

\section{Funding}

No funding was provided for this research. 


\section{References}

1 Brasil. Ministério da Saúde. Estratégia Nacional para o Enfrentamento da Hanseníase: 2019-2022. Brasília: Ministério da Saúde, 2020.

2 World Health Organization. Global leprosy (Hansen disease) update, 2019: time to step-up prevention initiatives. 2020 [cited 2020 Sep. 27]. Available from: https://www.who.int/publications-detail-redirect/who-wer9536 [Internet].

3 Brasil. Ministério da Saúde. Boletim Epidemiológico de Hanseníase 2020. Brasília: Ministério da Saúde, 2020. [cited 2020 Nov. 26]. Available from: http://www.aids.gov.br/pt-br/pub/2020/boletim-epidemiologico-de-hans eniase-2020 [Internet].

4 Brasil. Ministério da Saúde. Manual de prevenção de incapacidades. 2nd edn, Cadernos de prevenção e reabilitação em hanseníase, Brasília: Ministério da Saúde, 2008. Available from: http://bvsms.saude.gov.br/ bvs/publicacoes/manual_prevencao_incapacidades.pdf [Internet].

5 Husain S. Decompression of peripheral nerve trunks in leprosy prevents the development and progression of deformities? Indian J Lepr, 2013; 85(4): 163-169.

6 Kendall FP, McCreary EK, Provance PG, Rodgers MM, Romani WA. Músculos: Provas e Funções. 5th edn, Rio de Janeiro: Manole, 2007.

7 Buddingh H, Idle G. Grading impairment in leprosy. Lepr Rev, 2000; 71: 85-88.

8 Nienhuis WA, van Brakel WH, Butlin CR, van der Werf TS. Measuring impairment caused by leprosy: intertester reliability of the WHO disability grading system. Lepr Rev, 2004; 75(3): 221-232.

9 Brasil. Ministério da Saúde. Manual de reabilitação e cirurgia em hanseníase. 2nd edn, Cadernos de prevenção e reabilitação em hanseníase, Brasília: Ministério da Saúde, 2008. 148 p.

10 Mendes P, Telles C, Parise M, Acioly Marcus André. O papel da cirurgia descompressiva no tratamento da neuropatia em hanseníase. Rev Hosp Univ Pedro Ernesto, 2011.

11 Malaviya GN. Shall we continue with nerve trunk decompression in leprosy? Indian J Lepr, 2004; 76(4): 331-342.

12 Sette CS, Wachholz PA, Masuda PY, Figueira RBF da C, Filho MC, Soares CT et al. Surgical treatment of type I neuritis in a teenage boy with borderline tuberculoid leprosy. Rev Soc Bras Med Trop, 2015; 48(2): 228-230.

13 Balestrino A, Fiaschi P, Riccardi N, Camera M, Anania P, Martinoli C et al. Neurosurgical treatment of leprosy neuropathy in a low-incidence, European country. Neurol Sci Off J Ital Neurol Soc Ital Soc Clin Neurophysiol, 2019; 40(7): 1371-1375.

14 Castro LE, da Cunha AJLA, Fontana AP, de Castro Halfoun VLR, Gomes MK. Physical disability and social participation in patients affected by leprosy after discontinuation of multidrug therapy. Lepr Rev, 2014; 85(3): 208-217.

15 Croft RP, Nicholls PG, Steyerberg EW, Richardus JH, Cairns W, Smith S. A clinical prediction rule for nervefunction impairment in leprosy patients. Lancet, 2000; 355(9215): 1603-1606.

16 Rodrigues NC, Castro LE, Silva JG, Fontana AP, Neto BC, Sa VW et al. Physical disability and its social and functional repercussions in patients with leprosy after discharge from multidrug therapy. Lepr Rev, 2017; 88(1): 85-95.

17 Gonçalves SD, Sampaio RF, Antunes CM de F. Predictive factors of disability in patients with leprosy. Rev Saúde Pública, 2009; 43(2): 267-274.

18 Mantellini GG, Gonçalves A, Padovani CR. Incapacidades físicas em hanseníase: coisa do passado ou prioridade na prevenção? Hansen Int, 2009; 34(2): 33-39.

19 Reis FJJ. Limitação funcional e qualidade de vida de pacientes submetidos à neurólise para tratamento das neurites por hanseníase no HUCFF/UFRJ. Doutorado em Ciências, Rio de Janeiro: Universidade Federal do Rio de Janeiro - UFRJ, 2012.

20 Husain S, Kumar A, Yadav VS, Malaviya GN. Ulnar and median nerves in paucibacillary leprosy-a follow-up study of electrophysiological functions in patients before and after nerve trunk decompression. Lepr Rev, 2003; 74(4): 374-382.

21 Husain S, Mishra B, Prakash V, Malaviya GN. Results of surgical decompression of ulnar nerve in leprosy. Acta Leprol, 1998; 11(1): 17-20. 